\title{
Noise pollution: it's time to noise about
}

Sir,

Carrying the reputation of silent killer of 21st century, 'Noise' a modern day pollutant is defined as an unpleasant, disharmonic and undesired acoustic signal. World Health Organization (WHO) has estimated it dimensions and warned that $10 \%$ of the population is exposed to both short and long term health related issues due to noise. Whilst facing the conflict between development and human health, the noise favors the present day industrialization and urbanization. ${ }^{1}$ The harmful effect of noise may start from intrauterine life and continues till old age. Noise of more than $85-95 \mathrm{~dB}$ affects both the fetus and neonates by damaging the cochlear hair cell and results in high frequency hearing loss. At times prematurity, low birth rate and birth defects are also associated with exposure to high $\mathrm{dB}$ noise.

The functional acoustic-vestibular system develops around 26 to 28 weeks so is the development of the hearing capability in the fetus. In neonatal intensive care units (NICU), the permissible limit is less than $45 \mathrm{~dB}$ as suggested by American Academy of Pediatrics Committee (AAP). ${ }^{2}$ Children surrounded by noisy environment of indoor or outdoor origin develop stress, decreased learning capability, poor concentration, behavioral issues which altogether leads to a poor long term personality development. A sudden exposure to high $\mathrm{dB}$ noise even rupture the ear drum (acoustic trauma). The household use of music system, computer games and televisions and other highly noisy home appliances or gadgets should be discarded or exchanged without consideration of their costs because health is most valuable. $^{3}$

Due to routine outdoor activities adult population is much more affected with noise pollution and develop both auditory and non-auditory effects. Noise induced hearing loss (NIHL) which is a major cause of preventable hearing impairment develop with a long term exposure of more than $80-85 \mathrm{~dB}$ of sound. The irreversible damage of cochlear hair cells in organ of corti occur because of direct damage (acoustic trauma) or through inflammatory or immune mediated defense mechanism mediated by transforming growth factor-B (TGF-B). Cells which are present at the base of cochlea are more sensitive to high frequency noise. These basal cells are predominantly affected and are responsible for high frequency hearing loss. The role of TGF-B1 peptide inhibitor like P144 and may show promising results by improving the degenerative changes and the hearing threshold. Similarly omega 3 fatty acids supplementation may also be helpful in ameliorating the progress of hearing loss. Genes like NOX-3 and CDH-23 in susceptible individuals also contribute to NIHL. ${ }^{4,5}$ This NIHL is also commonly associated with tinnitus, hyperacusis and reduced ability to understand which leads to social and behavioral problems and even withdrawal from society.

Another long term impact of noise is disturbed sleep or insomnia. It has been found that REM phase of sleep is predominantly affected both with sleep disturbance and micro arousals. These people also face a difficulty to achieve and maintain a plateau for sufficient time duration which produces a cascade of pathophysiological changes responsible for increased heart rate and blood pressure, atherosclerosis, myocardial infarction (MI) or even stroke. ${ }^{6}$ This happens in individuals who are exposed to more than $60 \mathrm{~dB}$ of sound level for a reasonably longer duration. Chronic noise exposure to high $\mathrm{dB}$ also increases the incidence of diabetes mellitus in population. Development of stress due to continuous noise produces annoyance which triggers anger, exhaustion, displeasure and decreased sleep. ${ }^{7}$ The individual susceptibility towards noise is highly variable in individuals and the factors like smoking, type 2 diabetes mellitus, previous sensory neural hearing impairment and exposure to ototoxic drugs determines the susceptibility. Till date no medication has been proved to prevent or repair NIHL however high dose of magnesium prior or immediately after exposure of loud noise may have some beneficial effect. A high dose of corticosteroids is also found to be effective for acute acoustic trauma if instituted within three days.

The Supreme Court of India in the year 2005 gave a strict verdict on noise pollution and said that rigid norms of permissible sound level in urban and rural areas on residential, commercial or industrial grounds should be imposed. Simple priceless measures which could help in the longer run should be encouraged like avoiding honking, use of soundless fire crackers, use of speaker mode on the mobile phone for longer duration of conversations, encouraging the supervised use of earphones and use of personal sound protection devices like ear plugs etc. ${ }^{8}$ The need of the hour is to hit this health hazard by self and community discipline by implementing strict national policy to minimize its ill effects.

\section{Bhupendra Chaudhary ${ }^{1 *}$, Ansh Chaudhary ${ }^{2}$}

${ }^{1}$ Department of Medicine, Jaswant Rai Super Speciality Hospital, Meerut, Uttar Pradesh, India 
${ }^{2}$ Department of Medicine, Bharati Vidyapeeth Medical College and Research Centre, Pune, Maharashtra, India

*Correspondence:

Dr. Bhupendra Chaudhary, E-mail: doctorabp1567@gmail.com

\section{REFERENCES}

1. Passchier-Vermeer W, Passchier WF. Noise exposure and public health. Environment Health Perspectives. 2000 Mar;108(suppl 1):123-31.

2. Hohmann C, Grabenhenrich L, dekluizenaar Y, Tischer C, Heinrich J. Health effects of chronic noise exposure in pregnancy and childhood. Int $\mathrm{J}$ Environ Health. 2013;216:217-29.

3. Hetu R, Truchon-Gagnon, Bilodeau SA. Problems of noise in school settings: a review of literature and the results of an exploratory study. J Speech Lang Pathol Audiol. 1990;14:31-8.

4. Golz A, Westerman ST, Westerman LM, Goldenberg D, Netzer A, Wiedmyer T, et al. The effect of noise on the vestibular system. Am J Otolaryngol. 2001;22:190-6.

5. Kowalski JJ, Pawelczyk M, Rajkowska E. Genetic variants of $\mathrm{CDH} 23$ with noise induced hearing loss. Otoneurotol. 2014:35-7.

6. Joo EY, Yoon CW, Koo DL, Kim D, Hong SB. Adverse effects of 24 hours of sleep deprivation on cognition and stress hormones. J Clini Neurol. 2012 Jun 1;8(2):146-50.

7. Babisch W, Pershagen G, Selander J, Houthuijs D, Breugelmans O, Cadum E, et al. Noise annoyancea modifier of the association between noise level and cardiovascular health?. Sci Total Environmen. 2013 May 1;452:50-7.

8. Taljaard DS, Leishman NF, Eikelboom RH. Personal listening devices and the prevention of noise induced hearing loss in children: The Cheers for Ears Pilot Program. Noise Health. 2013-15:2618 .

Cite this article as: Chaudhary B, Chaudhary A. Noise pollution: it's time to noise about. Int J Adv Med 2020;7:1194-5. 